UVX 2010 (2011) 213-216

DOI: $10.1051 / \mathrm{uvx} / 2011031$

(C) Owned by the authors, published by EDP Sciences, 2011

\title{
Results obtained with high efficiency gratings for EUV application
}

\author{
Ph. Michallon ${ }^{1}$, B. Dalzotto ${ }^{1}$, C. Constancias ${ }^{1}$, J. Wallace ${ }^{2}$ and M. Saib ${ }^{3}$ \\ ${ }^{1}$ CEA Grenoble - MINATEC, 17 rue des Martyrs, 38054 Grenoble, France \\ 2 University of Wisconsin-Madison, Center for NanoTechnology, 425 Henry Mall, Suite 2130, \\ WI 53706, USA \\ ${ }^{3}$ CNRS - LTM Grenoble - MINATEC, 17 rue des Martyrs, 38054 Grenoble, France
}

\begin{abstract}
EUV lithography is planned to address the $22 \mathrm{~nm}$ node and beyond. Resolution limit for chemical amplified resists is one of the major issues for EUV lithography development. Currently, exposure studies concerning limit of resolution in resists are done in interferometer tools using synchrotron radiation light or EUV scanners. In the framework of a stand alone EUV interferometer development, due to the low power of EUV sources, we have studied and developed new gratings with high efficiency.

In this work, we developed gratings for EUV applications with a theoretical efficiency of $28 \%$ compared to gratings currently used in EUV interferometer ( $7 \%$ efficiency). Manufacturing process to realize $100 \mathrm{~nm}$ thick silicon membranes and gratings etched in molybdenum layer were developed. This high efficiency is a necessary step to build a successful standalone EUV interferometer. Membranes and gratings characteristics will be described. Exposure tests have been performed using synchrotron radiation. Results obtain with first and second order diffraction will be presented.
\end{abstract}

\section{INTRODUCTION}

EUV lithography is one of the best technologies in order to reach advanced nodes below $22 \mathrm{~nm}$ half pitch.

In this context, the resist availability is a fundamental step. Limit of resolution achievable by resists is one of the major interest points. To evaluate the capability of resists [1-3], a stand alone EUV interferometer could be very simple to use and it allows a fast assessment of the resist performances. We have carried out a study to design a stand alone interferometer capable to print interference lines with a pitch below $16 \mathrm{~nm}$. Due to the laboratory EUV source availability and their performances in terms of spatial coherency and power level, Leti has developed a new high efficiency grating.

Moreover, ultra thin silicon membranes, of sub micrometric thickness, are of a great interest for many applications like UV or RX spectrometry, NEMS or sensors. State of the art silicon technology fabrication using lithography, deposition, dry or wet etching allows generating structures in the nanometer range [4]. It has already been reported the fabrication of sub micrometric SiNx film for optical engineering [5]. Silicon thin film is of a great advantage compare to SiNx for the UV and X Ray domain because of its higher transparency: 100nm thick membrane of Si gives $85 \%$ transparency at $13.5 \mathrm{~nm}$ wavelength compare to $40 \%$ achieved with SiNx membrane.

One of the key points to print interference pattern in EUV-IL is the quality and the diffraction efficiency of the grating used for fringe patterning. More than the silicon membrane which support the grating, the highest diffraction efficiency can be achieved in EUV range with grating made with materials like Molybdenum or Niobium that give phase shift grating with low attenuation.

This is an Open Access article distributed under the terms of the Creative Commons Attribution-Noncommercial License 3.0, which permits unrestricted use, distribution, and reproduction in any noncommercial medium, provided the original work is properly cited. 
In this article we report results obtained in evaluation tests of the new high efficiency gratings using EUV synchrotron light.

Gratings are designed to achieve high diffraction efficiency in order to build stand alone EUV interferometer or to allow ultra high resolution patterning with the second order diffraction interferometry.

\section{MEMBRANE AND GRATING FABRICATION}

A patent pending is on the fabrication process of the Si membrane and Mo gratings so we only give here general information about grating and membrane. Stress materials on membrane and grating must be perfectly controlled in order to guarantee the integrity and the flatness level required range (1 $\mathrm{nm}$ variation for EUV interference application). We are able to make several millimeters side lengths membrane, typically $3 \times 3 \mathrm{~mm}^{2}$ to $10 \times 10 \mathrm{~mm}^{2}$, for thicknesses ranging from $100 \mathrm{~nm}$ to $200 \mathrm{~nm}$.

The membrane morphology and flatness have been measured with an optical and an interferometer microscope. -a shows pictures of the membranes, $3 \times 3 \mathrm{~mm}^{2}$. It is shown on $-\mathrm{b}$ that free-standing membrane is perfectly flat with deformation less than $1 \mathrm{~nm}$ on several millimetres wide.

(a)

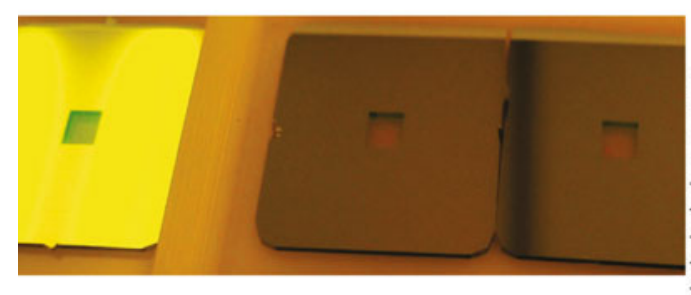

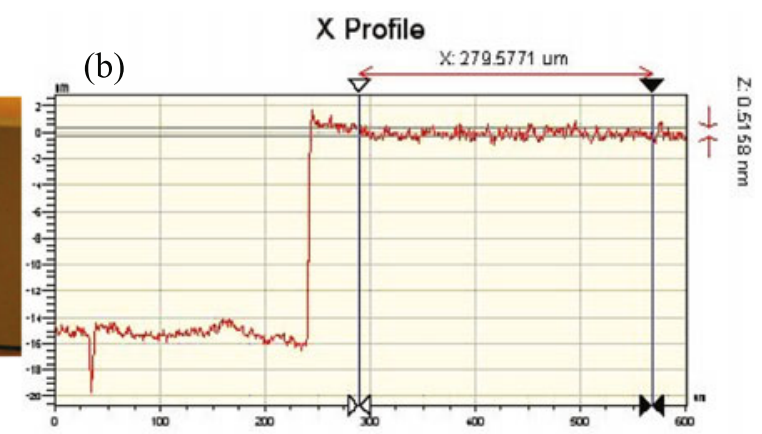

Figure 1. (a) Picture of the 8 inches wafers with $3 \times 3 \mathrm{~mm}^{2}$ Si membrane of $100 \mathrm{~nm}$ thick. (b) Si membrane profile of obtained by interferometer microscopy showing flatness better than $1 \mathrm{~nm}$.

Then, a thorough study allows us to determinate the optimum Mo layer deposition process to obtain the more adapted morphology Mo layer. On top of the membrane $85-90 \mathrm{~nm}$ thick Molybdenum is deposited by plasma sputtering with deposition conditions adjusted to reduce stress and film roughness.

Line and space are performed by Ebeam lithography using Gaussian Ebeam from Vistec. Iso-dense pitches down too $30 / 30 \mathrm{~nm}$ are patterned into a silicon membrane.

Lithography is transferred in molybdenum with several etching steps.

Each grating has typically $800 \mu \mathrm{m}$ length which allows the layout optimization for Ebeam exposure without more than one exposure field stitching to prevent from interference speckle as describe by M. Saidani [6].

Then due to the weak attenuation of Molybdenum at $13.5 \mathrm{~nm}$ wavelength a zero order blocker is required. The efficiency of this zero order must be sufficient to guarantee the good contrast on the first order interference lines printed.

\section{SI MEMBRANE AND MO GRATING TEST PRINTS ON SYNCHROTRON}

First grating manufactured in Leti clean room has been tested with EUV SRC synchrotron light at University of Wisconsin-Madison [3]. 


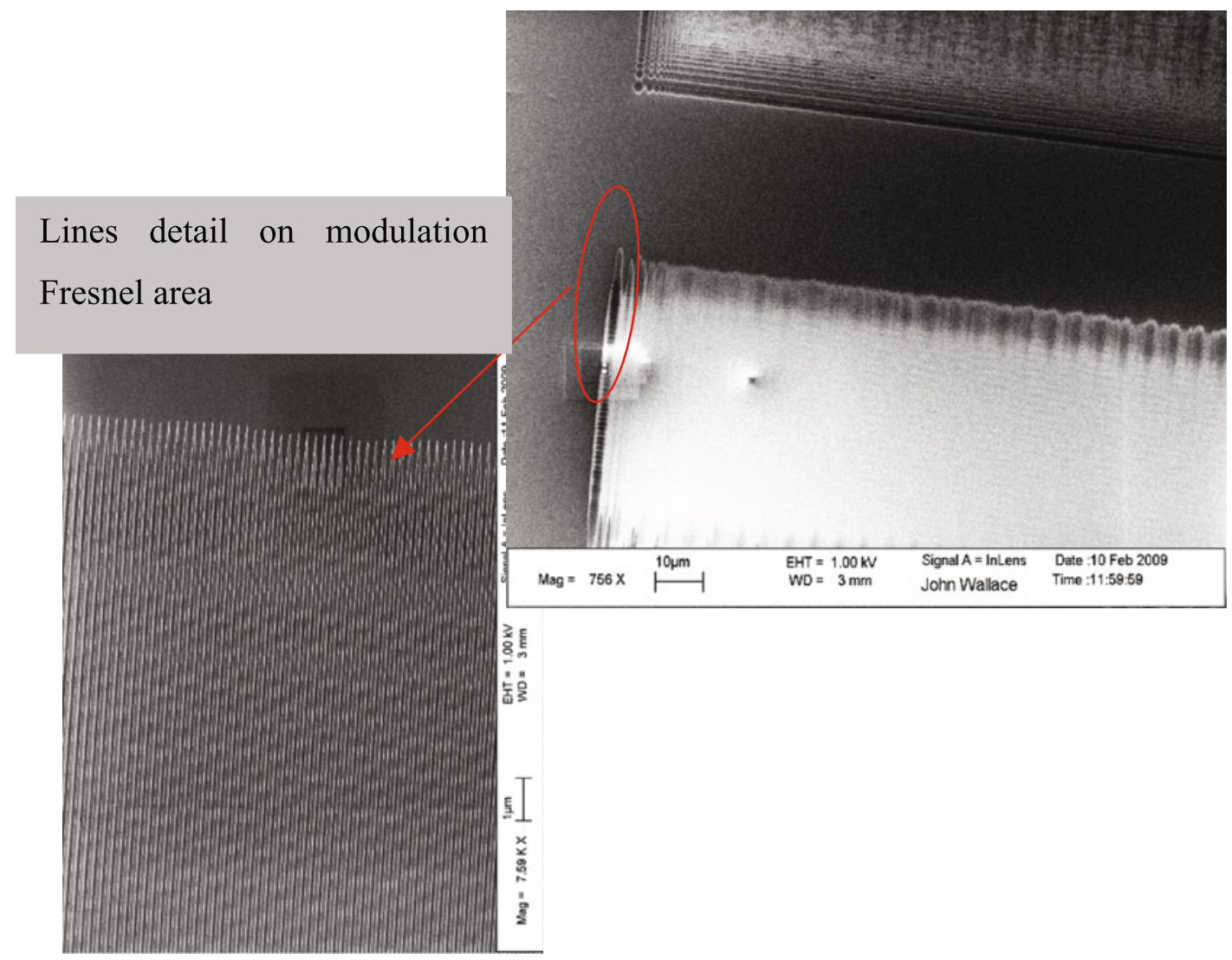

Figure 2. Fresnel perturbations and modulation on edge of grating.

Goal of the print tests is to evaluate the silicon membrane transmission and grating efficiency @ EUV $1=13.5 \mathrm{~nm}$. Print tests of 100, 35, 25, $20 \mathrm{~nm}$ half pitch in first order are performed with gratings of 200, 70, 50, $40 \mathrm{~nm}$ half pitch. The minimal size of grating width is evaluated by taking into account the impact of Fresnel perturbations on the edges of the gratings. This last point allows us to adapt the minimum width of spatial coherency needed for the EUV source.

The EUV flux within a bandwidth of $13.5 \pm 0.7 \mathrm{~nm}(92.5 \pm 5 \mathrm{eV})$ is first measured after a $\mathrm{Zr}$ filter over a $3 \times 3 \mathrm{~mm}^{2}$ windows with an AX100 photodiode. Then with the same synchrotron beam conditions, the $3 \times 3 \mathrm{~mm}^{2}$ Si membrane transmissions is measured. The photodiode current ratio gives a transmission of $86 \%$ for the $100 \mathrm{~nm}$ thick Si membrane. The same measurement is performed with a $100 \mathrm{~nm}$ thick Si3N4 membrane which gives $\mathbf{3 7 \%}$ transparency. In the same experimental condition, the Si membrane $+90 \mathrm{~nm}$ thick Mo layer gives a transparency of $42 \%$.

The diffraction efficiency of the "new" grating is compared, under same beam conditions, with a classical EUV grating (using membrane Si3N4 and Cr). For each grating, exposure times to access dose to clear on PMMA are evaluated. Exposure time with "classical" gratings obtained is around $12 \mathrm{~s}$ compared to $0.8 \mathrm{~s}$ needed for our gratings. This shows an efficiency improved by a factor of around 12 .

The diffraction efficiency is evaluated with respect to the resist thickness measurement after development for multiple exposure doses. In the linear range of the PMMA contrast curve, the thickness ratio is proportional to the energy ratio. The thickness ratio between the first order diffracted pattern and the background, out of grating transmission, i.e $100 \mathrm{~nm}$ thick $\mathrm{Si}+90 \mathrm{~nm}$ thick Mo, allows estimating 
the first order diffraction efficiency. The intensity profile transmitted through the membrane gives the PMMA profile. After development, with 1:3 MIBK: IPA for 3 minutes, for several exposure doses between $22 \mathrm{~mJ} / \mathrm{cm}^{2}$ to $95 \mathrm{~mJ} / \mathrm{cm}^{2}$, the PMMA thicknesses are measured along the resist profile with a KLA-Tencor HRP-profilometer. The thickness ratio in the linear range, i.e $30 \mathrm{~mJ} / \mathrm{cm}^{2}$ to $50 \mathrm{~mJ} / \mathrm{cm}^{2}$, gives the intensity ratio transmitted between first order diffraction and background. Taking into account the $42 \%$ transparency of the out of grating membrane we can estimate the absolute first order efficiency of the Mo grating to be $27 \%$. This experimental value is in good agreement with the calculated first order efficiency according to Ref [7]. With Mo grating of $90 \mathrm{~nm}$ thick with a gap period ratio of 50\% the calculation give a first order efficiency of $31 \%$, with a gap period ratio of $65 \%$ the calculation give a first order efficiency of $26 \%$.

The Fresnel perturbations (figure 2) due of the edge of the grating are measured. Several prints are performed by variation of the distance between gratings and wafer. The width of perturbation on edge of the first order print is measured. The width of Fresnel perturbations measured is 6 to $10 \mu \mathrm{m}$. The width of this perturbation is fixed by the size of gratings and this is independent of grating wafer distance.

\section{CONCLUSION}

We succeed in the fabrication of ultra-thin silicon membrane of $100 \mathrm{~nm}$ thick of several square millimetres with a planarity better than $1 \mathrm{~nm}$. The transparency of such Si membrane, at $13.5 \mathrm{~nm}$ wavelength, is greater than $85 \%$ compare to $42 \%$ achieved with usual Si3N4 membrane. We have also demonstrated the fabrication of Mo grating with a first order diffraction efficiency of $27 \%$ down to $40 \mathrm{~nm}$ L/S. Such grating could be used for EUV-IL with efficient exposure even at the second order diffraction for ultra high resolution resist patterning, down to $10 \mathrm{~nm}$ half pitch. Due to the low absorption of the Si membrane and the Mo background, i.e less than $58 \%$, a zero absorber layer has been patterned with a second lithography level aligned with respect to the first grating level.. Since $30 \mathrm{~nm}$ L/S grating on membrane and zero blocker with only $0.3 \%$ transmission have been developed. This will allow us to print in second order (access prints L/S below $10 \mathrm{~nm}$ ) and reduce considerably the specification constraints needed for the EUV source on stand-alone interferometer.

\section{Acknowledgments}

The authors would like to thank Paul Nealy, John Wallace for providing access to there EUV beam line interferometer setup at Madison Winsconsin SRC; especially for the availability and expertise for the measurements; E. Lajoinie and Y. Le Cunff for all etching engineering developed for the membrane far from the usual CMOS etching they have. This study is partly supported by the ANR 'EUVIL' funded by the french research ministry under contract number ANR-07-NANO-056.

\section{References}

[1] H. H. Solak et al., Appl. Phys. Lett. 75, 2328, (1999)

[2] H. H. Solak, W. Li, D. He, J. Wallace, and F. Cerrina AIP Conf. Proc. 52199 (2000)

[3] A. Isoyan, A. Wüest, J. Wallace, F. Jiang, and F. Cerrina Opt. Express 169106 (2008)

[4] M. E. Motamedi, Opt. Eng. 36, 1280 (1997)

[5] Chien Chieh Lee et al., Opt. Let. Vol. 28n No 14, Jul 15 (2003)

[6] M. Saidani, H.H. Solak, Microelectronic Engineering Vol; 86, 4-6 (April 2009)

[7] http://henke.lbl.gov/optical_constants/tgrat2.html 